\title{
Prototype-to-Style: Dialogue Generation with Style-Aware Editing on Retrieval Memory
}

\author{
Yixuan $\mathrm{Su}^{1}$, Yan Wang ${ }^{2}$, Simon Baker ${ }^{1}$, Deng $\mathrm{Cai}^{3}$, Xiaojiang $\mathrm{Liu}^{2}$, Anna Korhonen ${ }^{1}$, and Nigel Collier ${ }^{1}$ \\ ${ }^{1}$ University of Cambridge \\ ${ }^{2}$ Tencent AI Lab \\ ${ }^{3}$ The Chinese University of Hong Kong \\ \{ys484,sb895,alk23,nhc30\}@cam.ac.uk, thisisjcykcd@gmail.com \\ \{brandenwang,kieranliu\}@ tencent.com
}

\begin{abstract}
The ability of a dialog system to express prespecified language style during conversations has a direct, positive impact on its usability and on user satisfaction. We introduce a new prototype-to-style (PS) framework to tackle the challenge of stylistic dialogue generation. The framework uses an Information Retrieval (IR) system and extracts a response prototype from the retrieved response. A stylistic response generator then takes the prototype and the desired language style as model input to obtain a high-quality and stylistic response. To effectively train the proposed model, we propose a new style-aware learning objective as well as a de-noising learning strategy. Results on three benchmark datasets from two languages demonstrate that the proposed approach significantly outperforms existing baselines in both in-domain and crossdomain evaluations ${ }^{1}$.
\end{abstract}

\section{Introduction}

Most early research on dialogue response generation focused on generating grammatical and contextually relevant responses (Ritter et al., 2011; Chen et al., 2017; Martinovsky and Traum, 2003). While promising results have been demonstrated (Wen et al., 2016; Wang et al., 2016), syntactically coherent responses alone do not guarantee an engaging and attractive dialogue system. Expressing a unique and consistent speaking style has been shown to be crucial for increasing the user's engagement with dialogue systems (Gan et al., 2017). There are various definitions of language style (Roberts, 2003; Bell, 1984; Bell and Johnson, 1997; Niederhoffer and Pennebaker, 2002; Traugott, 1975). In this work, from a purely computational standpoint, we refer to language style as any characteristic style of expression. Hence,

\footnotetext{
${ }^{1}$ All code and trained models will be made publicly available.
}

our work is in line with previous work on dialogue generation with emotion (Zhou et al., 2018; Huang et al., 2018; Zhou and Wang, 2018; Zhong et al., 2019); response attitude (Niu and Bansal, 2018), and speaker personality ( $\mathrm{Li}$ et al., 2016b).

The aforementioned approaches explicitly incorporate the language style information into the model configuration either via embeddings or memory modules to control the process of response generation. In our replication experiments, we found that these approaches tend to overemphasise the importance of the language style. As a result, the generated responses tend to be generic and non-informative (Li et al., 2016a), but they do express a distinct style; e.g., they generate a generic response: "I am happy to hear that." that conveys a 'happy' emotion to different queries.

In this work, we propose a novel prototypeto-style (PS) framework to tackle the challenge of stylistic dialogue generation. Our motivation is two-fold: (1) Human-written responses are informative and diverse, which could be leveraged as guidance for the generation model; (2) However, the retrieved response is not guaranteed to express the desired language style. Moreover, the quality of the retrieved response varies among different queries due to the instability of the IR system. Therefore, to transform the retrieved result into a relevant and stylistic response, an adequate editing process is necessary.

An illustration of the proposed framework is shown in Figure 1, where a prototype is first extracted from the retrieved response. The stylistic response generator then takes the desired language style and the extracted prototype as additional input to obtain an adequate and stylistic response. The proposed stylistic response generator mainly inherits from the GPT-2 model (Radford et al., 2019) which is pre-trained with a large unlabeled text corpus. However, the GPT-2 model 


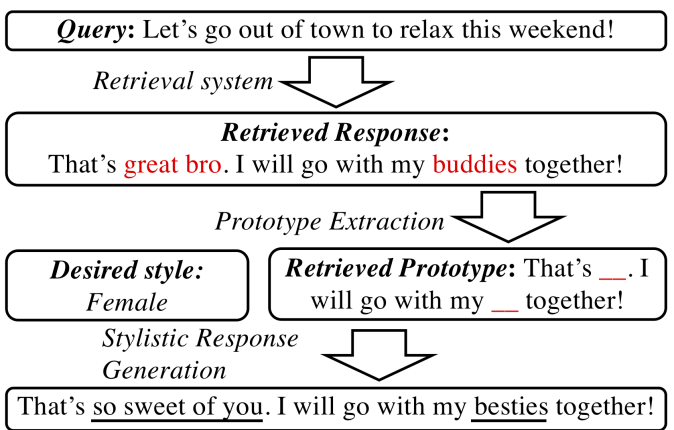

Figure 1: Prototype-to-Style Framework: It first constructs a neutral response prototype by masking the stylistic words from the retrieved response. The stylistic response generator then takes the extracted prototype and the desired language style information to generate an adequate and stylistic response.

does not naturally fit the task of dialogue generation. To this end, we design various adaptations to the model architecture to extend the GPT2 model to address the task of dialogue generation. Furthermore, in order to control the style of the generated responses, we train the model with a novel style-aware maximum likelihood estimation (MLE) objective that encodes additional style knowledge into the model's parameters. Finally, to mitigate the possible effect that the retrieved response containing irrelevant and inappropriate information with respect to the input query, we adopt a de-noising learning strategy (Jain and Seung, 2008; Krull et al., 2019) to prevent the model from uncritically copying the prototype.

To fully evaluate the proposed approach, we conduct extensive experiments on three benchmark datasets. Results of both human and automatic evaluation show that the proposed approach significantly outperforms several strong baselines. In addition, we also conduct an extensive crossdomain experiment to demonstrate that the proposed approach is more robust than such baselines.

It should be noted that stylistic dialogue generation is different from the task of text style transfer. Text style transfer aims to rewrite the input sentences such that they possess certain language styles, while rigorously preserving their semantic meaning (Jin et al., 2019). On the other hand, stylistic dialogue generation does not aim at preserving the semantic meaning of the input sentences. Instead, it aims at generating sentences that are adequate and relevant responses to the input sentences, while expressing the prespecified language styles.
In summary, the contributions of this work are: (1) We propose a novel framework that tackles the challenge of stylistic dialogue generation by leveraging useful information contained in the retrieved responses; (2) We propose a new stylistic response generator by making proper adaptations to a large-scale pre-trained language model. We train our model with a new style-aware learning objective in a de-noising manner. Experiments show that the proposed model outperforms many strong baselines on three benchmark datasets on both in-domain and cross-domain evaluations.

\section{Related Work}

We summarize three categories of relevant work in the following.

Text Style Transfer: The task of text style transfer aims to transfer the style contained in a sentence while preserving its meaning. Li et al. (2018) proposed a DRG framework to tackle this task with the help of external knowledge. Recently, based on the pre-trained language model, Sudhakar et al. (2019) further improved the system performance under the same DRG framework.

Retrieval Guided Dialogue Generation: Many prior works (Song et al., 2018; Zhu et al., 2019; Wu et al., 2019; Cai et al., 2019) proposed to leverage information from the retrieved responses to improve the system performance on non-task oriented dialogue generation. It should be noted that all these approaches aim to improve the content quality of the generated responses but do not take the style aspect into consideration.

Stylistic Dialogue Generation: Extensive research has tried to tackle the task of stylistic dialogue generation. $\mathrm{Li}$ et al. (2016b) proposed to represent the user's personality with embeddings and incorporated them into the decoder structure to control the response generation process. Niu and Bansal (2018) used reinforcement learning to train the generation model via the interaction with a pre-trained classifier to generate responses with specified attitude. Zhou et al. (2018); Huang et al. (2018); Zhou and Wang (2018); Zhong et al. (2019) incorporated external knowledge into the model architecture either via embeddings or internal and external memory modules, such that during the generation process, emotion-based styles can be dynamically controlled. Gao et al. (2019) 
proposed to use a shared latent space for stylistic dialogue generation.

\section{Methodology}

The proposed framework leverages the results acquired from an IR system, A major challenge is that the retrieved response is not guaranteed to express the desired language style. At the first step, a neutral response prototype is extracted by masking all stylistic words contained in the retrieved response. A stylistic response generator then takes the desired language style and the extracted prototype as additional input to generate an adequate and stylistic response to the input query. To better emphasize the generation of stylistic expressions, we propose a style-aware learning objective. Finally, to prevent the model from learning to uncritically copy the prototype, we adopt a denoising learning strategy (Jain and Seung, 2008; Krull et al., 2019) to train the generator.

\subsection{Prototype Extraction}

The response prototype is constructed from the retrieved response by masking the stylistic words. To determine whether a word is stylistic, we use the pointwise mutual information (PMI) (Church and Hanks, 1990) metric. The relevance between the word $x$ and the style $s$ is measured as

$$
\operatorname{PMI}(x ; s)=\log \frac{p(x, s)}{p(x) p(s)},
$$

where $p(x, s)$ is the frequency that the word $x$ appears in a response with style $s$ in the training corpus. And a word $x$ is stylistic given the style $s$ if $\operatorname{PMI}(x, s) \geq t_{s}$. In our experiments, we empirically set $t_{s}$ as $t_{s}=\frac{3}{4} \times \max _{v \in \mathcal{V}} \operatorname{PMI}(v ; s)$, where $\mathcal{V}$ is the vocabulary set of the training corpus. Given the set of all possible language styles $\mathcal{S}$, the stylistic vocabulary $\mathcal{S} \mathcal{V}$ is defined as all words that express any style $s \in \mathcal{S}$. An example is provided in Figure 1 where the prototype: "That's _. I will go with my _together!" is extracted from the retrieved response by masking the stylistic words great, bro and buddies.

\subsection{Stylistic Response Generator}

The proposed Stylistic Response Generator inherits from the GPT-2 (Radford et al., 2019) model which consists of a 12-layer decoder-only Transformer (Vaswani et al., 2017). To make use of the GPT-2 model, the input tokens must be a consecutive natural sequence (e.g. sentence, document).
Based on the input sequence, the input representation is constructed by adding up the token embeddings and the corresponding position embeddings.

To achieve the goal of adapting the GPT-2 model under the proposed PS framework, we first make modifications to the form of the input sequence. As shown in Figure 2, we construct the input sequence as the concatenation of the input query, the response prototype and the reference response. Then we introduce a special token $[B]$ to indicate the boundary between these three parts. To further ensure the model can identify the different parts of the input sequence, we introduce a new segment level input which consists of three learnable segment embeddings $E_{Q}, E_{P}$ and $E_{R}$ to indicate the positions of the input query, the response prototype and the response history.

To control the language style of the generated response, we propose to incorporate learnable style embeddings into the input representation. Specifically, we add the style embeddings ${ }^{2}$ to the entire part of the response history. This way, the model is constantly aware of the desired language style through the entire generation process.

\subsection{Learning}

\subsubsection{Style-Aware Learning Objective}

We propose to use a new style-aware learning objective to train the stylistic response generator. Consider a training instance consists of the input query $\mathbf{X}=\left(x_{1}, \ldots, x_{N}\right)$, the reference response $\mathbf{Y}=\left(y_{1}, \ldots, y_{T}\right)$, the reference language style $s$ and the response prototype $\mathbf{C}=\left(c_{1}, \ldots, c_{T}\right)$, the proposed objective is defined as

$$
\begin{aligned}
& L_{\mathrm{S}-\mathrm{MLE}}(\theta)= \\
& -\sum_{i=1}^{T} \log p_{\theta}\left(y_{i} \mid y_{1}, \ldots, y_{i-1} ; \mathbf{X}, \mathbf{C}, s\right) \cdot f\left(y_{i}\right) \\
& \quad f\left(y_{i}\right)= \begin{cases}1+\alpha & \text { if } y_{i} \in \mathcal{S} \mathcal{V} \\
1 & \text { otherwise, }\end{cases}
\end{aligned}
$$

where $\theta$ are the model parameters and $\mathcal{S V}$ is the stylistic vocabulary introduced in $\S 3.1$. By increasing $\alpha$, the proposed objective encodes more knowledge about stylistic expressions into the model parameters.

We find that including the language model as an auxiliary objective in addition to the supervised

\footnotetext{
${ }^{2}$ Each style embedding corresponds to one specific language style; e.g. if we consider three different gender styles, the number of different style embeddings is 3 .
} 


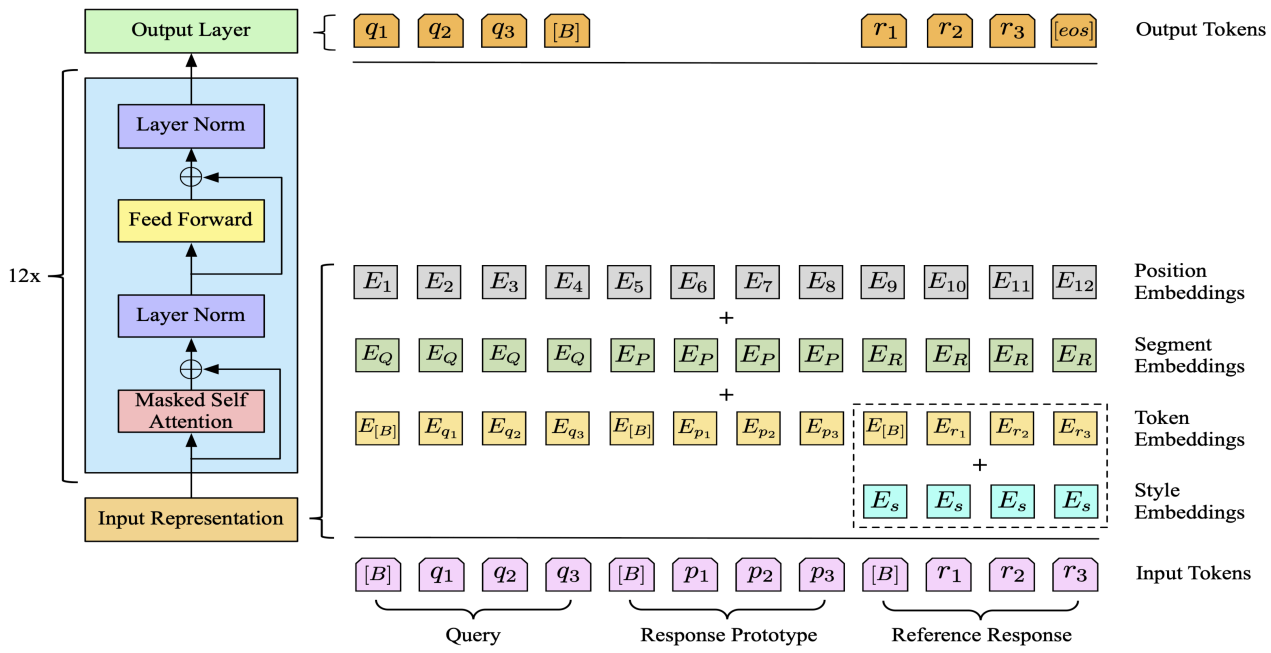

Figure 2: Illustration of the proposed Stylistic Response Generator: The input representation is constructed by adding up four different level embeddings. By specifying different style embeddings, the model can generate responses with different language styles.

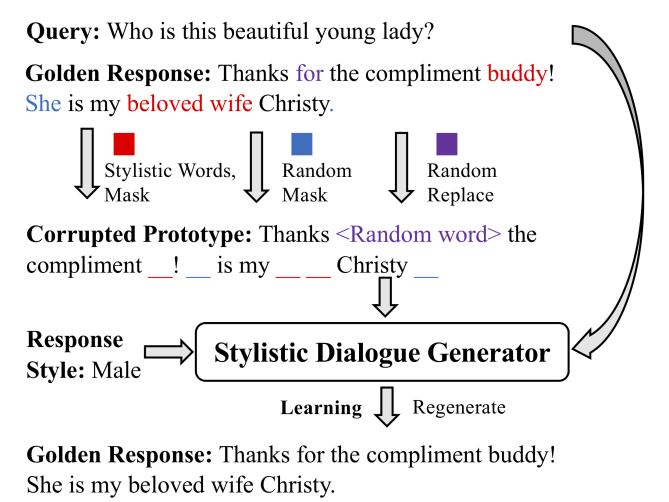

Figure 3: Illustration of de-noising training strategy.

style-aware learning objective helps to improve generalization as well as accelerate convergence. This observation is in line with Rei (2017); Radford et al. (2018). In this work, the language model objective is defined as the reconstruction loss of the input query based on itself:

$$
\begin{aligned}
L_{\mathrm{LM}}(\theta) & =-\log p_{\theta}(\mathbf{X}) \\
& =-\sum_{j=2}^{N} \log p_{\theta}\left(x_{j} \mid x_{1}, \ldots, x_{j-1}\right) .
\end{aligned}
$$

The final learning objective is then defined as

$$
L(\theta)=L_{\mathrm{S}-\mathrm{MLE}}(\theta)+\beta L_{\mathrm{LM}}(\theta),
$$

where $\beta$ regulates the importance of the auxiliary objective $^{3}$.

\footnotetext{
${ }^{3}$ The $\alpha$ in $L_{\mathrm{S}-\mathrm{MLE}}(\theta)$ is set to be 0.2 and the $\beta$ is set to be 1.0
}

\subsubsection{De-noising Training}

We use a de-noising training strategy similar to Jain and Seung (2008); Krull et al. (2019) for training data construction, as shown in Figure 3. Specifically, during training, the response prototype is extracted from the reference response by the following steps. First, we mask all the stylistic words in the reference response. Second, we randomly select some words (40\%) and replace it with a special token [MASK] or a random word drawn from the vocabulary.

The second step is necessary otherwise the model will learn to generate a response by uncritically copying the response prototype, since the prototype after the first step is always an integral part of the golden response. This copy mechanism is undesirable since during testing the retrieved response is likely to contain information that is irrelevant to the input query. Thus, we deliberately train the response generator with noisy input to let the model learn to filter out the inappropriate information contained in the response prototype.

\section{Datasets}

We conduct extensive experiments on three dialogue datasets: gender-specific (Chinese) dataset, emotion-specific (Chinese) dataset, and sentimentspecific (English) dataset. For each dataset, we randomly select 200 instances as a held-out test set for evaluation. 


\begin{tabular}{|c|c|c|c|}
\hline Queries & \multicolumn{2}{|c|}{$26,265,224$} & Percentage(\%) \\
\hline \multirow{3}{*}{ Responses } & Positive & $4,275,978$ & $16.28 \%$ \\
\cline { 2 - 4 } & Negative & $6,282,641$ & $23.92 \%$ \\
\cline { 2 - 4 } & Neutral & $15,706,605$ & $59.80 \%$ \\
\hline
\end{tabular}

Table 1: Data Statistic of Sentiment-Specific Dataset

\subsection{Gender-Specific Dialogue Dataset}

We use a publicly available gender-specific dialogue dataset (Su et al., 2020). In this dataset, each response contains one specific gender preference including Female, Male and Neutral.

\subsection{Emotion-Specific Dialogue Dataset}

We use a publicly available emotion-specific dataset (Zhou et al., 2018) which contains responses with 6 different emotions including Like, Disgust, Happy, Anger, Sad and Other.

\subsection{Sentiment-Specific Dialogue Dataset}

To construct this dataset, we first build a classifier on the basis of BERT (Devlin et al., 2019) and finetuned it on the the SemEval-2017 Subtask A dataset (Rosenthal et al., 2017). This dataset consists of twitter instances with different sentiments including Positive, Negative and Neutral.

The sentiment classifier attains $81.4 \%$ classification accuracy which is further used to annotate the OpenSubtitles dataset (Lison and Tiedemann, 2016). The data statistic of the resulting sentimentspecific dialogue dataset is shown in Table 1.

\section{Experiments}

\subsection{Pretraining and Implementation Details}

As there is no off-the-shelf pre-trained word-level language model in Chinese, we manually pretrained one. The corpus collection and model pretraining details are presented in the supplementary material. For the English pre-trained language model, we use the PyTorch adaptation released by the HuggingFace team ${ }^{4}$.

To optimize the model, we use the Adam optimizer (Kingma and Ba, 2015) with a batch size of 64 and learning rate of 2e-5. During inference, the retrieval system is built from the training corpus, and the retrieved responses are selected using the Jaccard similarity (Lipkus, 1999) between queries.

During the inference stage, we retrieve the candidates from the training set. Specifically, we employ Jacquard Similarity to calculate the similarity

\footnotetext{
${ }^{4}$ https://github.com/huggingface/pytorch-openaitransformer-lm
}

between the input query q and queries in training set and find the most similar query $\mathrm{q}^{\prime}$. Then we directly adopt the response of the retrieved query $\mathrm{q}^{\prime}$ to construct the response prototype.

\subsection{Model Comparison}

We compare the proposed approach with several competitive baselines that can be categorized into two classes: generative approaches and retrievalbased approaches.

\subsubsection{Generative Approaches}

Seq2seq: Standard sequence-to-sequence model with attention mechanism (Bahdanau et al., 2015; Luong et al., 2015).

GPT2-FT: To examine the effect of leveraging the pre-trained language model for the task of dialogue generation, we directly fine-tune the GPT-2 model on the dialogue data without any designed adaptations.

Speaker: Model proposed by Li et al. (2016b) which incorporates distributed style embeddings into the structure of decoding cells to control the generation process.

ECM: Model proposed by Zhou et al. (2018) which uses memory modules to control the stylistic expressions in the generated responses.

\subsubsection{Retrieval-Based Approaches}

Skeleton-to-Response (SR): Model proposed by Cai et al. (2019) which modifies the retrieved response based on the lexical difference between the input and the retrieved query. This approach does not take the style aspect into consideration.

Retrieval + Style Transfer (RST): For this approach, we apply the state-of-the-art style transfer (Sudhakar et al., 2019) model on the retrieved response. This approach does not consider the input query information during the transfer process.

Retrieval + Reranking (RRe): Given the input query, a style classifier is used to rerank the top 10 retrieved responses. The response with the highest score on the desired style is selected.

\subsubsection{Ablation Study}

PS: The full model proposed in this work.

PS w/o R: In the ablated model, we examine how the retrieved prototype effects our model's performance. To this end, we remove the response prototype from the input representation. 


\begin{tabular}{|c|c|c|c|c|c|c|c|c|c|c|}
\hline \multirow{2}{*}{ Style } & \multirow{2}{*}{ Metrics } & \multicolumn{4}{|c|}{ Generative } & \multicolumn{3}{|c|}{ Retrieval-Based } & \multicolumn{2}{|c|}{ Ours } \\
\hline & & Seq2seq & GPT2-FT & Speaker & ECM & SR & RST & $\mathrm{RRe}$ & PS w/o R & PS \\
\hline \multirow{3}{*}{ Male } & Quality $\uparrow$ & 2.97 & 3.33 & 2.49 & 2.56 & 2.58 & 2.15 & 2.78 & 2.94 & 3.48 \\
\hline & Style Expression $\uparrow$ & 2.93 & 2.99 & 3.51 & 3.60 & 2.98 & 3.21 & 3.01 & 3.36 & 3.75 \\
\hline & Ranking $\downarrow$ & 3.04 & 2.71 & 3.42 & 3.15 & 3.89 & 4.01 & 3.43 & 2.34 & 1.56 \\
\hline \multirow{3}{*}{ Female } & Quality $\uparrow$ & 2.97 & 3.31 & 2.86 & 2.81 & 2.60 & 2.16 & 3.11 & 3.01 & 3.42 \\
\hline & Style Expression $\uparrow$ & 3.07 & 3.02 & 3.01 & 3.09 & 3.02 & 3.14 & 3.09 & 3.49 & 3.64 \\
\hline & Ranking $\downarrow$ & 2.94 & 2.62 & 3.18 & 3.20 & 3.66 & 3.86 & 2.89 & 2.28 & 1.52 \\
\hline \multirow{5}{*}{ Overall } & Quality $\uparrow$ & 2.98 & 3.32 & 2.68 & 2.67 & 2.59 & 2.14 & 2.94 & 2.98 & 3.45 \\
\hline & Style Expression $\uparrow$ & 3.00 & 3.05 & 3.26 & 3.35 & 3.03 & 3.17 & 3.01 & 3.43 & 3.69 \\
\hline & Ranking $\downarrow$ & 2.99 & 2.66 & 3.30 & 3.17 & 3.78 & 3.94 & 3.16 & 2.31 & 1.54 \\
\hline & Distinct-1(\%)个 & 27.64 & 36.42 & 26.15 & 12.45 & 37.62 & 33.12 & $48.52 \dagger$ & 29.98 & 40.88 \\
\hline & Distinct-2(\%)个 & 72.33 & 74.30 & 50.40 & 31.64 & 84.33 & 85.63 & $94.11 \dagger$ & 78.54 & 90.82 \\
\hline
\end{tabular}

Table 2: Evaluation Results on Gender-Specific Dialogue Generation: $\uparrow$ means the higher the better and $\downarrow$ means the lower the better, bold font denotes the best scores for each metric. Sign tests on evaluation scores show that the proposed model significantly outperforms other models with p-value $<0.05$ with the only exception marked by $\dagger$.

\begin{tabular}{|c|c|c|c|c|c|c|c|c|c|c|}
\hline \multirow{2}{*}{ Style } & \multirow{2}{*}{ Metrics } & \multicolumn{4}{|c|}{ Generative } & \multicolumn{3}{|c|}{ Retrieval-Based } & \multicolumn{2}{|c|}{ Ours } \\
\hline & & Seq2seq & GPT2-FT & Speaker & ECM & SR & RST & $\mathrm{RRe}$ & PS w/o R & PS \\
\hline \multirow{3}{*}{ Like } & Quality $\uparrow$ & 3.06 & 3.48 & 2.62 & 2.61 & 2.49 & 2.25 & 2.61 & 3.49 & 3.62 \\
\hline & Style Expression $\uparrow$ & 3.01 & 3.05 & $3.95 \dagger$ & $4.38 \dagger$ & 2.99 & 3.25 & 2.83 & $3.93 \dagger$ & 3.77 \\
\hline & Ranking $\downarrow$ & 3.77 & 3.53 & 3.47 & 2.95 & 4.71 & 4.43 & 4.56 & 2.11 & 1.86 \\
\hline \multirow{3}{*}{ Disgust } & Quality $\uparrow$ & 3.03 & $3.47 \dagger$ & 2.07 & 1.99 & 2.45 & 2.34 & 2.58 & 3.27 & 3.41 \\
\hline & Style Expression $\uparrow$ & 2.53 & 2.68 & $4.06 \dagger$ & $3.97 \dagger$ & 2.85 & 3.17 & 2.99 & 3.39 & 3.61 \\
\hline & Ranking $\downarrow$ & 3.95 & 3.51 & 3.79 & 3.97 & 4.45 & 3.95 & 4.11 & 2.15 & 1.85 \\
\hline \multirow{3}{*}{ Нарру } & Quality $\uparrow$ & 3.03 & 3.48 & 2.06 & 2.46 & 2.51 & 2.43 & 2.69 & 3.51 & 3.68 \\
\hline & Style Expression $\uparrow$ & 4.06 & 3.49 & $4.83 \dagger$ & $4.94 \dagger$ & 3.09 & $4.73 \dagger$ & 2.91 & $4.81 \dagger$ & 4.59 \\
\hline & Ranking $\downarrow$ & 3.44 & 3.91 & 4.19 & 3.32 & 5.51 & 3.31 & 5.35 & 1.85 & 1.62 \\
\hline \multirow{3}{*}{ Anger } & Quality $\uparrow$ & 2.98 & $3.43 \dagger$ & 1.94 & 1.95 & 2.41 & 2.31 & 2.66 & 3.01 & 3.37 \\
\hline & Style Expression $\uparrow$ & 1.76 & 2.35 & $3.93 \dagger$ & $4.02 \dagger$ & 2.77 & 3.46 & 2.94 & $3.82 \dagger$ & 3.83 \\
\hline & Ranking $\downarrow$ & 5.27 & 4.05 & 4.21 & 3.97 & 4.71 & 3.75 & 4.05 & 2.20 & 1.78 \\
\hline \multirow{3}{*}{ Sad } & Quality $\uparrow$ & 2.95 & $3.44 \dagger$ & 2.14 & 2.09 & 2.37 & 2.30 & 2.59 & 3.12 & 3.42 \\
\hline & Style Expression $\uparrow$ & 1.83 & 2.36 & $3.64 \dagger$ & 3.43 & 2.77 & 3.24 & 2.92 & $3.68 \dagger$ & 3.58 \\
\hline & Ranking $\downarrow$ & 5.01 & 3.81 & 4.01 & 4.18 & 4.47 & 3.85 & 3.97 & 1.98 & 1.81 \\
\hline \multirow{5}{*}{ Overall } & Quality $\uparrow$ & 3.01 & $3.46 \dagger$ & 2.16 & 2.22 & 2.44 & 2.33 & 2.63 & 3.31 & 3.46 \\
\hline & Style Expression $\uparrow$ & 2.64 & 2.79 & $4.08 \dagger$ & $4.14 \dagger$ & 2.89 & 3.57 & 2.92 & $3.93 \dagger$ & 3.85 \\
\hline & Ranking $\downarrow$ & 4.29 & 3.74 & 3.94 & 3.68 & 4.77 & 3.86 & 4.41 & 2.06 & 1.78 \\
\hline & Distinct-1(\%)个 & 13.61 & 19.37 & 8.99 & 5.85 & 31.71 & 30.39 & $44.67 \dagger$ & 22.55 & 36.47 \\
\hline & 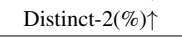 & 34.03 & 59.65 & 21.08 & 15.68 & 78.91 & 82.41 & $92.58 \dagger$ & 69.29 & 87.48 \\
\hline
\end{tabular}

Table 3: Evaluation Results on Emotional-Specific Dialogue Generation

\subsection{Evaluation Metrics}

The quality of dialogue responses is known to be difficult to measure automatically (Deriu et al., 2019); we therefore rely on human evaluation. To evaluate the responses, we hire five annotators from a commercial annotation company. To prevent introducing potential bias to the annotators, all results are randomly shuffled before being evaluated. All results are evaluated by the annotators following the metrics below.

Quality: This metric evaluates the content quality of the generated responses. The annotators are asked to give a score within 5-point scale where 5 means perfectly human-like response (relevant, fluent and informative), 3 means marginally acceptable and 1 means unreadable and impossible to understand.
Style Expression: This metric measures how well the generated responses express the desired style. The annotators give a score ranging from 1 to 5 to this metric, where 5 means very strong style, 3 means no obvious style and 1 means very conflicted style. The style conflict means the generated style is conflicted to the desired one (e.g. female to male, positive to negative emotion).

Ranking: The annotators are further asked to jointly evaluate the content quality and the style expression of the generated responses from different approaches. Then the annotators give a ranking to each result where top 1 means the best ${ }^{5}$.

\subsection{Main Results}

Both human and automatic evaluation results on the three benchmark datasets are shown in Table

\footnotetext{
${ }^{5}$ The same ranking is allowed to be assigned to results from different approaches if they have the same overall quality.
} 


\begin{tabular}{|c|c|c|c|c|c|c|c|c|c|c|}
\hline \multirow{2}{*}{ Style } & \multirow{2}{*}{ Metrics } & \multicolumn{4}{|c|}{ Generative } & \multicolumn{3}{|c|}{ Retrieval-Based } & \multicolumn{2}{|c|}{ Ours } \\
\hline & & Seq2seq & GPT2-FT & Speaker & ECM & SR & RST & $\mathrm{RRe}$ & PS w/o R & PS \\
\hline \multirow{3}{*}{ Positive } & Quality $\uparrow$ & 2.63 & 2.97 & 2.72 & 2.72 & 1.90 & 2.42 & 2.49 & 2.93 & 3.28 \\
\hline & Style Expression $\uparrow$ & 2.52 & 2.55 & 3.51 & 3.89 $\dagger$ & 2.72 & 2.96 & 2.70 & 3.44 & 3.76 \\
\hline & Ranking $\downarrow$ & 4.39 & 4.05 & 3.10 & 2.38 & 4.71 & 4.10 & 4.12 & 2.61 & 1.79 \\
\hline \multirow{3}{*}{ Negative } & Quality $\uparrow$ & 2.69 & 2.96 & 2.99 & 2.56 & 1.82 & 2.26 & 2.64 & 2.80 & 3.20 \\
\hline & Style Expression $\uparrow$ & 3.15 & 3.09 & $3.62 \dagger$ & 3.47 & 2.71 & 3.18 & 2.82 & 3.42 & 3.63 \\
\hline & Ranking $\downarrow$ & 3.62 & 3.68 & 3.48 & 3.04 & 4.81 & 4.00 & 3.80 & 2.78 & 2.39 \\
\hline \multirow{5}{*}{ Overall } & Quality $\uparrow$ & 2.66 & 2.97 & 2.86 & 2.64 & 1.86 & 2.34 & 2.57 & 2.87 & 3.24 \\
\hline & Style Expression $\uparrow$ & 2.83 & 2.82 & 3.57 & $3.68 \dagger$ & 2.72 & 3.07 & 2.76 & 3.43 & 3.70 \\
\hline & Ranking $\downarrow$ & 4.00 & 3.85 & 2.79 & 2.71 & 4.76 & 4.05 & 3.96 & 2.69 & 2.09 \\
\hline & Distinct-1(\%)个 & 24.65 & 29.92 & 23.61 & 14.22 & 30.06 & 40.13 & $49.94 \dagger$ & 32.29 & 44.70 \\
\hline & Distinct-2(\%)^ & 48.74 & 56.27 & 43.11 & 23.72 & 75.73 & 71.73 & $91.59 \dagger$ & 68.35 & 87.15 \\
\hline
\end{tabular}

Table 4: Evaluation Results on Sentiment-Specific Dialogue Generation

2, 3 and 4. For each dataset, we present results on individual styles as well as the overall results.

We observe that the proposed model achieves the top performance results on most of the metrics. It generates responses with both intense style and high response quality. In addition, we also measure the diversity of the generated responses with two automatic metrics: Distinct-1 and Distinct-2 (Li et al., 2016b). The results show that the proposed model achieves the closest performance to that of the RRe approach whose responses are all written by human. On the ranking metric which jointly evaluates the content quality and the style expression, the proposed model outperforms other approaches by a substantial margin.

From the results in Table 3 and 4, we can observe that ECM obtains the highest style expression scores on the emotion and sentiment dialogue datasets. This is because ECM directly incorporates the style information into its model architecture to force the generation of stylistic expressions. However, as shown in the quality scores, this behavior also undermines the quality of the generated responses. Therefore, the overall performance of ECM is not optimal as shown in the results of the ranking metric.

From the experiment results, we observe that removing retrieved information (PS w/o R) from the proposed model causes a drastic drop on the quality score. This demonstrates that the retrieved information is indispensable for the model to generate a stylistic response and maintain a high response quality. In addition, comparing with GPT2FT baseline, the ablated model (PS w/o R) shows similar content quality and much stronger stylistic expression, which is gained from the model architectural design and the new training strategy.

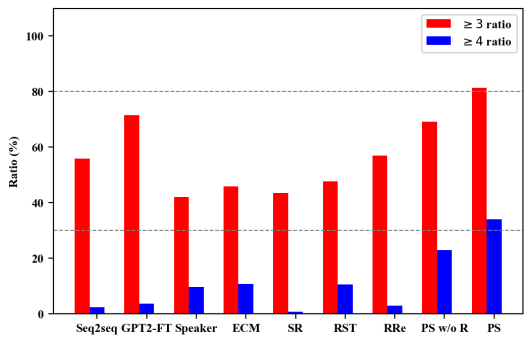

Figure 4: Balance between Quality and Style: The $\geq 3$ ratio means the ratio of responses whose both scores are greater or equal to 3 ; $\geq 4$-ratio means the ratio of responses whose both scores are greater or equal to 4 .

\subsection{Further Analysis}

We present further discussions and empirical analysis of the proposed approach.

\subsubsection{Balance between Quality and Style}

In practice, a satisfactory stylistic dialogue system should express the desired style on the premise of the response quality. Based on the criterion of human evaluation metric, 3 is the marginal score of acceptance. So we deem a response as marginally acceptable by actual users when both quality and style expression scores are greater or equal to 3 . On the other hand, 4 is the score that well satisfies the users, so responses with both scores greater or equal to 4 are deemed as satisfying to actual users.

The ratios of both scores $\geq 3$ and $\geq 4$ are shown in Figure 4, from which we can see that the proposed approach outperforms all other approaches on $\geq 3$-ratio and $\geq 4$-ratio. The proposed model best balances the trade-off between the response quality and style expression and therefore generating most acceptable and satisfying responses.

\subsubsection{Cross-Domain Evaluation}

To evaluate the robustness of different approaches, we further analyze their performances when there is a notable difference between the data distribu- 


\begin{tabular}{|c|c|c|c|c|}
\hline Query & Retrieved Response & Style & Generated Response & Translation \\
\hline \multirow{7}{*}{$\begin{array}{l}\text { 没有做完的梦最痛。 } \\
\text { Unfinished dreams } \\
\text { hurt the most. }\end{array}$} & \multirow{7}{*}{$\begin{array}{l}\text { 这几天有时候做咞梦。 } \\
\text { I somettimes have } \\
\text { nightmares at these days. }\end{array}$} & Male & 哥这几天一直都在做哥梦。 & Buddy, I have been having nightmares at these days. \\
\hline & & Female & 大姨妈来前几天我老做哥梦。 & I always have nightmares on the days before my menstruation. \\
\hline & & Like & 这几天我很享受做歌梦。 & I enjoy my nightmares at these days. \\
\hline & & Disgust & 最近我就烦的很，天天做㕵梦。 & I am so annoyed because I have nightmares everyday. \\
\hline & & Happy & 哈哈。这几天我经常梦到你。 & Lol. I always see you in dreams at these days. \\
\hline & & Anger & 靠！我要去死！这几天我老是做咞梦。 & $\mathrm{F}^{* *} \mathrm{k}$ ! I am going to die. I have too many nightmares at these days. \\
\hline & & Sad & 唉，日子没法过了，老是做㶾梦。 & Sigh, too many nightmares, I can't live with it anymore. \\
\hline & & & & \\
\hline \multirow{7}{*}{$\begin{array}{c}\text { 不辛苦!一切为 } \\
\text { 了民币! } \\
\text { Not tired at all! } \\
\text { Everything is for } \\
\text { money! }\end{array}$} & \multirow{7}{*}{$\begin{array}{c}\text { 人们想涨点工资。 } \\
\text { People want to get } \\
\text { a higher salary. }\end{array}$} & Male & 兄弟赶快给爷多涨点工资。 & Buddy, just hurry up and give your bro a higher salary. \\
\hline & & Female & 希望给我们男朋友多涨点工资。 & I hope our boyfriends can get higher salaries. \\
\hline & & Like & 真希望这次他们多涨点工资。 & I truly hope they can get higher salaries this time. \\
\hline & & Disgust & 鄙视他们！给他们少点工资。 & They are so contemptible! Their salary should be lower. \\
\hline & & Happy & 哈哈, 我会给他们多涨点工资! & Lol, I will give them higher salaries! \\
\hline & & Anger & 操，我不给他们涨工资。 & $\mathrm{F}^{* *} \mathrm{k}$ ! I will not raise their salaries. \\
\hline & & Sad & 可怜的孩子，给他多涨点工资吧。 & What a poor child, please give him a higher salary. \\
\hline
\end{tabular}

Table 5: Examples of generated responses with different gender and emotion styles. The words in red color are the informative details that the model extracts from the retrieved response.

\begin{tabular}{|l|l|l|l|}
\hline \multicolumn{1}{|c|}{ Query } & \multicolumn{1}{c|}{ Retrieved Response } & \multicolumn{1}{c|}{ Positive Sentiment } & \multicolumn{1}{c|}{ Negative Sentiment } \\
\hline I know a game perfect for the two of us. & You want us to target these old ones ? & I want us to target these pretty nice ones. & I don 't want us to target the wrong ones anymore. \\
\hline My answer would still be the same as before. & He can make his own decision. & You can make a pretty good decision. & You can make a very stupid decision. \\
\hline I don 't believe a word of this. & They all sound the same to me. & I mean it all sound pretty nice to me. & Damn all sound like shit to me! \\
\hline
\end{tabular}

Table 6: Examples of generated responses with different sentiments.

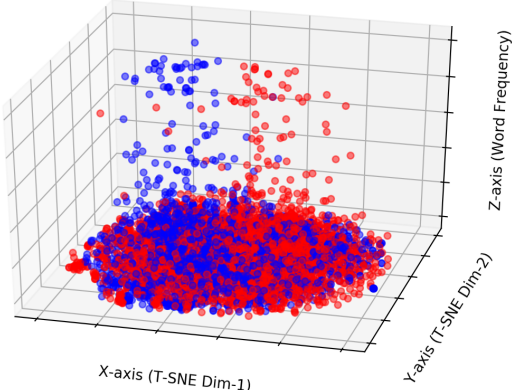

Figure 5: Blue and red dots represent the words in gender-specific and emotion-specific dataset. Each word $w_{d}$ is denoted as $\left(x_{d}, y_{d}, z_{d}\right)$ where $\left(x_{d}, y_{d}\right)$ is TSNE representation of its pretrained Glove embeddings (Pennington et al., 2014) and $z_{d}$ is the word frequency in the corresponding dataset. A notable distribution discrepancy between two domains can be observed.

tion of the training and testing set. Specifically, we use the models trained on gender-specific dataset to conduct inference on the test set of emotionspecific dataset and vise versa, which is regarded as domain variation. In Figure 5, we show the data distributions of these two datasets from which we can observe a notable distribution discrepancy. For evaluation, all results are evaluated with the same metrics as in the previous experiments. The averages response quality scores before and after domain variation are shown in Figure $6^{6}$. For a direct comparison, the in-domain performance of each model can be found in Table 2 and 3 .

As shown in Figure 6, some of the strong base-

\footnotetext{
${ }^{6}$ The concrete numerical results of cross-domain evaluation are shown in the supplementary material.
}

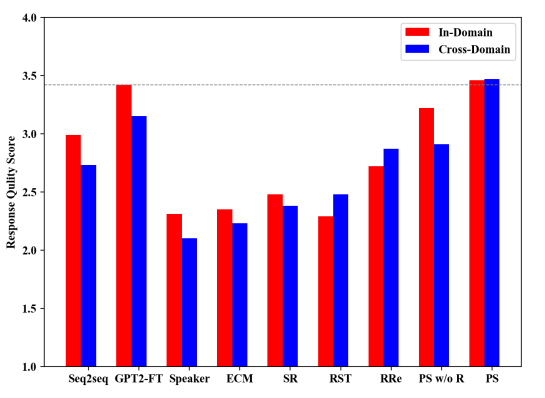

Figure 6: In-domain and cross-domain evaluations on the quality of generated responses. The red column represents the averaged quality score on in-domain test set, and the blue column denotes the averaged quality score after domain variation

lines exhibit a drastic drop in response quality after domain variation such as GPT2-FT and PS w/o R. In contrast, the PS model successfully maintains high response quality in spite of domain variation. The model seems to benefit from leveraging retrieved results to bridge the gap between the two different domains. This can also be observed in the results of RST and RRe which also use the retrieved results and get a even higher performance when facing domain variation.

\subsection{Case Study}

We present several examples of generated responses by the proposed PS approach. Table 5 shows responses with different gender and emotion styles, and Table 6 shows responses with different sentiments. Examples in Table 5 show that the proposed approach is able to extract in- 
formative details such as "have nightmares" and "higher salary" that are relevant to the queries from the retrieved responses. By taking the desired style as input, the proposed model generates adequate and stylistic responses while producing the informative details. Examples in Table 6 also demonstrate that the proposed model is able to generate responses with desired sentiments based on the informative details (e.g. " - want us to target _ones _,, “_can make _decision.” and “_ sound _ to me _.") contained in the retrieved response.

\section{Conclusion}

In this work, we propose a novel PS framework to tackle the task of stylistic dialogue generation. Additionally, we propose a new stylistic response generator which works coherently with the proposed framework. We conduct extensive experiments on three benchmark datasets from two languages. Results of human and automatic evaluation show that the proposed approach outperforms many strong baselines by a substantial margin.

\section{References}

Dzmitry Bahdanau, Kyunghyun Cho, and Yoshua Bengio. 2015. Neural machine translation by jointly learning to align and translate. In ICLR 2015, San Diego, CA, USA, May 7-9, 2015, Conference Track Proceedings.

Allan Bell. 1984. Language style as audience design. Language in Society, 13(2):145204.

Allan Bell and Gary Johnson. 1997. Towards a sociolinguistics of style. University of Pennsylvania Working Papers in Linguistics, 4(1):2.

Deng Cai, Yan Wang, Wei Bi, Zhaopeng Tu, Xiaojiang Liu, Wai Lam, and Shuming Shi. 2019. Skeletonto-response: Dialogue generation guided by retrieval memory. In Proceedings of the 2019 Conference of the North American Chapter of the Association for Computational Linguistics: Human Language Technologies, NAACL-HLT 2019, Minneapolis, MN, USA, June 2-7, 2019, Volume 1 (Long and Short Papers), pages 1219-1228.

Hongshen Chen, Xiaorui Liu, Dawei Yin, and Jiliang Tang. 2017. A survey on dialogue systems: Recent advances and new frontiers. SIGKDD Explorations, 19(2):25-35.

Kenneth Ward Church and Patrick Hanks. 1990. Word association norms, mutual information, and lexicography. Computational linguistics, 16(1):22-29.

Jan Deriu, Álvaro Rodrigo, Arantxa Otegi, Guillermo Echegoyen, Sophie Rosset, Eneko Agirre, and Mark
Cieliebak. 2019. Survey on evaluation methods for dialogue systems. CoRR, abs/1905.04071.

Jacob Devlin, Ming-Wei Chang, Kenton Lee, and Kristina Toutanova. 2019. BERT: pre-training of deep bidirectional transformers for language understanding. In Proceedings of the 2019 Conference of the North American Chapter of the Association for Computational Linguistics: Human Language Technologies, NAACL-HLT 2019, Minneapolis, MN, USA, June 2-7, 2019, Volume 1 (Long and Short Papers), pages 4171-4186.

Chuang Gan, Zhe Gan, Xiaodong He, Jianfeng Gao, and Li Deng. 2017. Stylenet: Generating attractive visual captions with styles. In 2017 IEEE Conference on Computer Vision and Pattern Recognition, CVPR 2017, Honolulu, HI, USA, July 21-26, 2017, pages 955-964.

Xiang Gao, Yizhe Zhang, Sungjin Lee, Michel Galley, Chris Brockett, Jianfeng Gao, and Bill Dolan. 2019. Structuring latent spaces for stylized response generation. In Proceedings of the 2019 Conference on Empirical Methods in Natural Language Processing and the 9th International Joint Conference on Natural Language Processing, EMNLP-IJCNLP 2019, Hong Kong, China, November 3-7, 2019, pages 1814-1823.

Chenyang Huang, Osmar R. Zaïane, Amine Trabelsi, and Nouha Dziri. 2018. Automatic dialogue generation with expressed emotions. In NAACL-HLT 2018, New Orleans, Louisiana, USA, June 1-6, 2018, Volume 2 (Short Papers), pages 49-54.

Viren Jain and H. Sebastian Seung. 2008. Natural image denoising with convolutional networks. In Advances in Neural Information Processing Systems 21, Proceedings of the Twenty-Second Annual Conference on Neural Information Processing Systems, Vancouver, British Columbia, Canada, December 8-11, 2008, pages 769-776.

Zhijing Jin, Di Jin, Jonas Mueller, Nicholas Matthews, and Enrico Santus. 2019. Unsupervised text style transfer via iterative matching and translation. CoRR, abs/1901.11333.

Diederik P. Kingma and Jimmy Ba. 2015. Adam: A method for stochastic optimization. In ICLR 2015, San Diego, CA, USA, May 7-9, 2015, Conference Track Proceedings.

Alexander Krull, Tim-Oliver Buchholz, and Florian Jug. 2019. Noise2void - learning denoising from single noisy images. In IEEE Conference on Computer Vision and Pattern Recognition, CVPR 2019, Long Beach, CA, USA, June 16-20, 2019 , pages 2129-2137.

Jiwei Li, Michel Galley, Chris Brockett, Jianfeng Gao, and Bill Dolan. 2016a. A diversity-promoting objective function for neural conversation models. In NAACL HLT 2016, The 2016 Conference of 
the North American Chapter of the Association for Computational Linguistics: Human Language Technologies, San Diego California, USA, June 12-17, 2016, pages 110-119.

Jiwei Li, Michel Galley, Chris Brockett, Georgios P. Spithourakis, Jianfeng Gao, and William B. Dolan. 2016b. A persona-based neural conversation model. In Proceedings of the 54th Annual Meeting of the Association for Computational Linguistics, ACL 2016, August 7-12, 2016, Berlin, Germany, Volume 1: Long Papers.

Juncen Li, Robin Jia, He He, and Percy Liang. 2018. Delete, retrieve, generate: a simple approach to sentiment and style transfer. In Proceedings of the 2018 Conference of the North American Chapter of the Association for Computational Linguistics: Human Language Technologies, NAACL-HLT 2018, New Orleans, Louisiana, USA, June 1-6, 2018, Volume 1 (Long Papers), pages 1865-1874.

Alan H Lipkus. 1999. A proof of the triangle inequality for the tanimoto distance. Journal of Mathematical Chemistry, 26(1-3):263-265.

Pierre Lison and Jörg Tiedemann. 2016. Opensubtitles2016: Extracting large parallel corpora from movie and TV subtitles. In Proceedings of the Tenth International Conference on Language Resources and Evaluation LREC 2016, Portorož, Slovenia, May 23-28, 2016.

Thang Luong, Hieu Pham, and Christopher D. Manning. 2015. Effective approaches to attentionbased neural machine translation. In EMNLP 2015, Lisbon, Portugal, September 17-21, 2015, pages 1412-1421.

Bilyana Martinovsky and David Traum. 2003. 2003.the error is the clue: Breakdown in humanmachine interaction. In In Proceedings of the ISCA Workshop on Error Handling in Dialogue Systems.

Kate Niederhoffer and James Pennebaker. 2002. Linguistic style matching in social interaction. Journal of Language and Social Psychology, 21:337-360.

Tong Niu and Mohit Bansal. 2018. Polite dialogue generation without parallel data. TACL, 6:373-389.

Jeffrey Pennington, Richard Socher, and Christopher D. Manning. 2014. Glove: Global vectors for word representation. In Proceedings of the 2014 Conference on Empirical Methods in Natural Language Processing, EMNLP 2014, October 25-29, 2014, Doha, Qatar, A meeting of SIGDAT, a Special Interest Group of the ACL, pages 1532-1543.

Alec Radford, Karthik Narasimhan, Tim Salimans, and Ilya Sutskever. 2018. Improving language understanding by generative pre-training. In Technical report, OpenAI.
Alec Radford, Jeffrey Wu, Rewon Child, David Luan, Dario Amodei, and Ilya Sutskever. 2019. Language models are unsupervised multitask learners.

Marek Rei. 2017. Semi-supervised multitask learning for sequence labeling. In Proceedings of the 55th Annual Meeting of the Association for Computational Linguistics, ACL 2017, Vancouver, Canada, July 30 - August 4, Volume 1: Long Papers, pages 2121-2130.

Alan Ritter, Colin Cherry, and William B. Dolan. 2011. Data-driven response generation in social media. In EMNLP 2011, 27-31 July 2011, John McIntyre Conference Centre, Edinburgh, UK, pages 583-593.

Julie Roberts. 2003. Style and sociolinguistic variation. American Anthropologist, 105.

Sara Rosenthal, Noura Farra, and Preslav Nakov. 2017. SemEval-2017 task 4: Sentiment analysis in twitter. In Proceedings of the 11th International Workshop on Semantic Evaluation (SemEval-2017).

Yiping Song, Cheng-Te Li, Jian-Yun Nie, Ming Zhang, Dongyan Zhao, and Rui Yan. 2018. An ensemble of retrieval-based and generation-based humancomputer conversation systems. In Proceedings of the Twenty-Seventh International Joint Conference on Artificial Intelligence, IJCAI 2018, July 13-19, 2018, Stockholm, Sweden, pages 4382-4388.

Yixuan Su, Deng Cai, Yan Wang, Simon Baker, Anna Korhonen, Nigel Collier, and Xiaojiang Liu. 2020. Stylistic dialogue generation viainformation-guided reinforcement learning strategy. arXiv preprint.

Akhilesh Sudhakar, Bhargav Upadhyay, and Arjun Maheswaran. 2019. Transforming delete, retrieve, generate approach for controlled text style transfer. arXiv preprint, abs/1908.09368.

Elizabeth Closs Traugott. 1975. William labov, sociolinguistic patterns. (conduct and communication, 4.) philadelphia: University of pennsylvania press, 1972. Language in Society, 4(1):89107.

Ashish Vaswani, Noam Shazeer, Niki Parmar, Jakob Uszkoreit, Llion Jones, Aidan N. Gomez, Lukasz Kaiser, and Illia Polosukhin. 2017. Attention is all you need. In Advances in Neural Information Processing Systems 30: Annual Conference on Neural Information Processing Systems 2017, 4-9 December 2017, Long Beach, CA, USA, pages 5998-6008.

Longyue Wang, Xiaojun Zhang, Zhaopeng Tu, Hang $\mathrm{Li}$, and Qun Liu. 2016. Dropped pronoun generation for dialogue machine translation. In ICASSP 2016, Shanghai, China, March 20-25, 2016, pages 6110 6114.

Tsung-Hsien Wen, Milica Gasic, Nikola Mrksic, Lina Maria Rojas-Barahona, Pei-Hao Su, Stefan Ultes, David Vandyke, and Steve J. Young. 2016. Conditional generation and snapshot learning in 
neural dialogue systems. In EMNLP 2016, Austin, Texas, USA, November 1-4, 2016, pages 21532162.

Yu Wu, Furu Wei, Shaohan Huang, Yunli Wang, Zhoujun Li, and Ming Zhou. 2019. Response generation by context-aware prototype editing. In The Thirty-Third AAAI Conference on Artificial Intelligence, AAAI 2019, The Thirty-First Innovative Applications of Artificial Intelligence Conference, IAAI 2019, The Ninth AAAI Symposium on Educational Advances in Artificial Intelligence, EAAI 2019, Honolulu, Hawaii, USA, January 27 - February 1, 2019, pages 7281-7288.

Peixiang Zhong, Di Wang, and Chunyan Miao. 2019. An affect-rich neural conversational model with biased attention and weighted cross-entropy loss. In AAAI 2019, Honolulu, Hawaii, USA, January 27 February 1, 2019., pages 7492-7500.

Hao Zhou, Minlie Huang, Tianyang Zhang, Xiaoyan Zhu, and Bing Liu. 2018. Emotional chatting machine: Emotional conversation generation with internal and external memory. In AAAI-18, New Orleans, Louisiana, USA, February $\overline{2-7,2018 \text {, pages }}$ 730-739.

Xianda Zhou and William Yang Wang. 2018. Mojitalk: Generating emotional responses at scale. In ACL 2018, Melbourne, Australia, July 15-20, 2018, Volume 1: Long Papers, pages 1128-1137.

Qingfu Zhu, Lei Cui, Weinan Zhang, Furu Wei, and Ting Liu. 2019. Retrieval-enhanced adversarial training for neural response generation. In Proceedings of the 57th Conference of the Association for Computational Linguistics, ACL 2019, Florence, Italy, July 28- August 2, 2019, Volume 1: Long Papers, pages 3763-3773. 
1 Dataset

Since there is no off-the-shelf dialogue dataset which specifically considers the gender style of the responses. To facilitate future research in this area, we manually collected over 4.5 million query-response pairs from popular Chinese social media platforms, including Tieba, Zhidao, Douban and Weibo. From the collected dataset, we randomly select a subset of 200,000 query-response pairs and recruit five professional annotators from an annotation company to annotate the gender style contained in the responses. For each query-response pair, each annotator is asked to assign one of the three labels (Male, Female or Neutral) to the response. Labels are selected if at least four out of five annotators agree.

The final annotated subset contains 5,184 male instances and 10,710 female instances. To proper balance the data distribution, we then randomly select 15,000 neutral instances. Therefore, the final gender style response classification dataset contains 5,184 male, 10,710 female and 15,000 neutral instances. Some examples of the annotated dataset are shown in Table 1.

\begin{tabular}{|c|l|l|}
\hline Style & \multicolumn{1}{|c|}{ Input Query } & \multicolumn{1}{c|}{ Response } \\
\hline \multirow{2}{*}{ Male } & 我们谁先来? & 我是單士, 当然请你先来。 \\
\cline { 2 - 3 } & Which one of us should come first? & I am a gentlemen, of course it should be you. \\
\hline \multirow{2}{*}{ Female } & 你多少岁? & 我是一个17岁的少女。 \\
\cline { 2 - 3 } & How old are you? & I am a 17-year old teenage girl. \\
\hline \multirow{2}{*}{ Neutral } & 我要找个人。 & 你是在找我吗? \\
\cline { 2 - 3 } & I am looking for someone. & Are you looking for me? \\
\hline
\end{tabular}

Table 1: Examples of gender response classification dataset : Both Chinese and translated versions are provided.

After building the gender classification dataset, we train a gender classifier based on BERT (?) which achieves a $91.7 \%$ accuracy. Then we use the trained classifier to automatically annotate the collected 4.5 million query-response pairs to get a large gender-specific dialogue dataset. The data statistic of the gender-style dialogue dataset is shown in Table 2. To facilitate future research in this area, both the gender classification dataset and the large gender-specific dialogue dataset will be made publicly available.

\begin{tabular}{|c|c|c|c|}
\hline Queries & \multicolumn{2}{|c|}{$4,579,712$} & Percentage(\%) \\
\hline \multirow{3}{*}{ Responses } & Male & 68,350 & $1.49 \%$ \\
\cline { 2 - 4 } & Female & 206,654 & $4.51 \%$ \\
\cline { 2 - 4 } & Neutral & $4,304,708$ & $94.00 \%$ \\
\hline
\end{tabular}

Table 2: Data Statistic of Gender-Specific Dialogue Dataset

\subsection{Emotion-Specific Dataset}

In addition to the gender-specific dataset, we use a publicly available emotion-specific dataset (?) which is also written in Chinese. This dataset contains responses with six different emotions including Like, Disgust, Happy, Anger, Sad and Other. We refer the readers to the original paper for more details of this dataset.

\subsection{Sentiment-Sepcific Dataset}

To evaluate the proposed model's performance across different languages, we also conduct experiments on a English sentiment-specific dialogue dataset. Specifically, we build the sentiment-specific dataset on the basis of the publicly available OpenSubtitles ${ }^{1}$ dataset (?).

To train a sentiment classifier, we resort to the SemEval-2017 Subtask A dataset (?), which consists of twitter instances with different sentiments (Positive, Negative and Neutral). The sentiment classifier is also constructed with BERT which achieves $71.4 \%$ classification accuracy. The trained classifier is further used to annotate the OpenSubtitles dataset and the resulting data statistic is shown in Table 3. 
ACL 2020 Submission ***. Confidential Review Copy. DO NOT DISTRIBUTE.

\begin{tabular}{|c|c|c|c|}
\hline Queries & \multicolumn{2}{|c|}{$26,265,224$} & Percentage(\%) \\
\hline \multirow{3}{*}{ Responses } & Positive & $4,275,978$ & $16.28 \%$ \\
\cline { 2 - 4 } & Negative & $6,282,641$ & $23.92 \%$ \\
\cline { 2 - 4 } & Neutral & $15,706,605$ & $59.80 \%$ \\
\hline
\end{tabular}

Table 3: Data Statistic of Sentiment-Specific Dialogue Dataset

\section{Experiments}

\subsection{Chinese Large-Scale Language Model Pre-training}

Because there is no off-the-shelf pre-trained word-level language model in Chinese, we manually pretrained one ourselves. Specifically, we first collected a large-scale Chinese corpus from popular Chinese News sites, including Sina, Baidu, Tencent, Toutiao, BBC China and New York Times China. We preprocess the acquired corpus with PKUSEG (?) tokenizer to create a word-level corpus. After filtering out invalid contents (e.g. URLs), the resulting corpus contains over 7.6 million sentences and over 350 million words.

Then we build the transformer-based language model following the same configuration as the one of ?. We refer the readers to the original paper for more details. We pre-train our language model for 10 epochs with 4 GeForce GTX 1080 Ti GPUs.

\subsection{Implementation Details}

For experiments on different datasets, we limit the vocabulary size as 20,000. To optimize the proposed model, we use Adam (?) optimizer with a batch size of 64 and learning rate of 2e-5. For each dataset, the model is trained for 3 epochs.

At inference stage, for simplicity, we build the retrieval system based on the training corpus. Specifically, given a new input query, the Jaccard similarity (?) is measured between the new input query and queries contained in the training corpus. Then we select the response of the most similar query in the training corpus as the retrieved response.

\section{Cross-Domain Evaluation}

In this section, we present evaluation results of different models when facing the domain variation. To this end, we use the model trained on gender-specific dataset to conduct inference on the test set of the emotion-specific dataset and vice versa. The results are evaluated by the annotators following the same protocol as the one in previous experiments. The numerical results of cross-domain evaluation are shown in Table 4 and 5.

\begin{tabular}{|c|c|c|c|c|c|c|c|c|c|c|}
\hline \multirow{2}{*}{ Style } & \multirow{2}{*}{ Metrics } & \multicolumn{4}{|c|}{ Generative } & \multicolumn{2}{c|}{ Retrieval-Based } & \multicolumn{3}{c|}{ Ours } \\
\cline { 3 - 11 } & & Seq2seq & GPT2-FT & Speaker & ECM & SR & RST & RRe & PS w/o R & PS \\
\hline \hline \multirow{3}{*}{ Male } & Quality $\uparrow$ & 2.72 & 3.13 & 2.17 & 2.43 & 2.26 & 2.22 & 2.49 & 3.02 & $\mathbf{3 . 4 0}$ \\
& Style Expression $\uparrow$ & 3.01 & 2.99 & 3.90 & 3.72 & 2.99 & 3.28 & 2.99 & 3.61 & $\mathbf{3 . 7 9}$ \\
& Ranking $\downarrow$ & 3.56 & 3.29 & 3.56 & 3.36 & 4.41 & 3.88 & 4.10 & 2.17 & $\mathbf{1 . 6 4}$ \\
\hline \multirow{3}{*}{ Female } & Quality $\uparrow$ & 2.74 & 3.15 & 2.76 & 2.54 & 2.28 & 2.20 & 2.54 & 2.93 & $\mathbf{3 . 2 3}$ \\
& Style Expression $\uparrow$ & 2.99 & 3.03 & 2.97 & 3.22 & 3.01 & 3.20 & 3.04 & 3.56 & $\mathbf{3 . 9 1}$ \\
& Ranking $\downarrow$ & 3.26 & 2.90 & 3.27 & 3.33 & 3.98 & 3.65 & 3.63 & 2.09 & $\mathbf{1 . 6 0}$ \\
\hline \multirow{3}{*}{ Overall } & Quality $\uparrow$ & 2.73 & 3.14 & 2.46 & 2.49 & 2.27 & 2.21 & 2.52 & 2.98 & $\mathbf{3 . 3 1}$ \\
& Style Expression $\uparrow$ & 3.00 & 3.01 & 3.44 & 3.48 & 3.00 & 3.24 & 2.99 & 3.59 & $\mathbf{3 . 8 5}$ \\
& Ranking $\downarrow$ & 3.41 & 3.09 & 3.42 & 3.34 & 4.20 & 3.76 & 3.87 & 2.13 & $\mathbf{1 . 6 2}$ \\
\hline
\end{tabular}

Table 4: Cross-Domain Evaluation Results on Gender-Specific Dialogue Generation: ( $\uparrow$ means the higher the better and $\downarrow$ means the lower the better. The best results of each metric are presented in bond font.) Sign tests on human evaluation scores show that our full model significantly outperforms other models with $\mathrm{p}$-value $<0.05$.

We also compare the results of both in-domain and cross-domain evaluations. The results for quality, style expression and ranking are shown in Figure 1,2 and 3 respectively.

Firstly, in Figure 1, drastic drop after domain-variation can be found on some strong baselines like GPT2-FT and PS w/o R. In contrast, the PS model successfully maintains high response quality after domain variation. It is benefit from the leveraging of retrieved results which helps to bridge the gap 
ACL 2020 Submission ${ }^{\star \star \star}$. Confidential Review Copy. DO NOT DISTRIBUTE.

\begin{tabular}{|c|c|c|c|c|c|c|c|c|c|c|}
\hline \multirow{2}{*}{ Style } & \multirow{2}{*}{ Metrics } & \multicolumn{4}{|c|}{ Generative } & \multicolumn{3}{c|}{ Retrieval-Based } & \multicolumn{2}{c|}{ Ours } \\
\cline { 3 - 10 } & & Seq2seq & GPT2-FT & Speaker & ECM & SR & RST & RRe & PS w/o R & PS \\
\hline \hline \multirow{3}{*}{ Like } & Quality $\uparrow$ & 2.75 & 3.17 & 2.11 & 2.30 & 2.44 & 2.57 & 3.03 & 3.10 & $\mathbf{3 . 6 1}$ \\
& Style Expression $\uparrow$ & 2.74 & 2.92 & $3.89 \dagger$ & $\mathbf{4 . 3 2} \dagger$ & 3.03 & 3.19 & 2.88 & $3.76 \dagger$ & 3.71 \\
& Ranking $\downarrow$ & 4.26 & 4.03 & 4.08 & 3.19 & 4.37 & 3.76 & 3.69 & 2.27 & $\mathbf{1 . 8 1}$ \\
\hline \multirow{3}{*}{ Disgust } & Quality $\uparrow$ & 2.76 & 3.18 & 1.99 & 2.10 & 2.46 & 2.52 & 3.18 & 2.88 & $\mathbf{3 . 6 1}$ \\
& Style Expression $\uparrow$ & 2.63 & 2.67 & $\mathbf{3 . 9 6} \dagger$ & $3.78 \dagger$ & 2.90 & 3.10 & 2.93 & $3.50 \dagger$ & 3.46 \\
& Ranking $\downarrow$ & 4.30 & 4.18 & 3.93 & 4.00 & 4.23 & 3.74 & 3.21 & 2.56 & $\mathbf{1 . 7 9}$ \\
\hline \multirow{3}{*}{ Happy } & Quality $\uparrow$ & 2.70 & 3.15 & 1.78 & 2.23 & 2.41 & 2.63 & 2.93 & 3.04 & $\mathbf{3 . 5 0}$ \\
& Style Expression $\uparrow$ & 3.97 & 3.41 & $4.84 \dagger$ & $\mathbf{4 . 9 5} \dagger$ & 3.03 & 4.63 & 2.91 & $4.80 \dagger$ & 4.70 \\
& Ranking $\downarrow$ & 3.88 & 4.48 & 4.39 & 3.42 & 5.41 & 2.95 & 4.76 & 1.98 & $\mathbf{1 . 5 7}$ \\
\hline \multirow{3}{*}{ Angeryyyyyyyyyy} & Quality $\uparrow$ & 2.72 & 3.17 & 1.83 & 1.84 & 2.41 & 2.61 & 3.09 & 2.54 & $\mathbf{3 . 5 1}$ \\
& Style Expression $\uparrow$ & 1.80 & 2.35 & $3.94 \dagger$ & $\mathbf{4 . 0 3} \dagger$ & 2.94 & 3.46 & 2.91 & $3.92 \dagger$ & 3.81 \\
& Ranking $\downarrow$ & 5.48 & 4.76 & 4.37 & 4.20 & 4.40 & 3.34 & 3.48 & 2.64 & $\mathbf{1 . 7 1}$ \\
\hline \multirow{3}{*}{ Sad } & Quality $\uparrow$ & 2.69 & 3.15 & 2.04 & 2.19 & 2.39 & 2.60 & 2.84 & 2.85 & $\mathbf{3 . 4 5}$ \\
& Style Expression $\uparrow$ & 1.99 & 2.44 & $\mathbf{3 . 6 2} \dagger$ & 3.32 & 2.86 & 3.14 & 2.96 & 3.56 & $\mathbf{3 . 6 1}$ \\
& Ranking $\downarrow$ & 4.96 & 4.24 & 4.15 & 4.06 & 4.10 & 3.34 & 3.45 & 2.31 & $\mathbf{1 . 6 7}$ \\
\hline \multirow{3}{*}{ Overall } & Quality $\uparrow$ & 2.73 & 3.16 & 1.95 & 2.13 & 2.42 & 2.59 & 3.01 & 2.88 & $\mathbf{3 . 5 4}$ \\
& Style Expression $\uparrow$ & 2.63 & 2.76 & $4.05 \dagger$ & $\mathbf{4 . 0 8} \dagger$ & 2.95 & 3.50 & 2.92 & $3.91 \dagger$ & 3.86 \\
& Ranking $\downarrow$ & 4.59 & 4.34 & 4.18 & 3.77 & 4.50 & 3.43 & 3.72 & 2.35 & $\mathbf{1 . 7 1}$ \\
\hline
\end{tabular}

Table 5: Cross-Domain Evaluation Results on Emotional-Specific Dialogue Generation: ( $\uparrow$ means the higher the better and $\downarrow$ means the lower the better. The best results of each metric are presented in bond font.) Sign tests on human evaluation scores show that our full model significantly outperforms other models with p-value $<0.05$ with the only exception marked by $\dagger$.

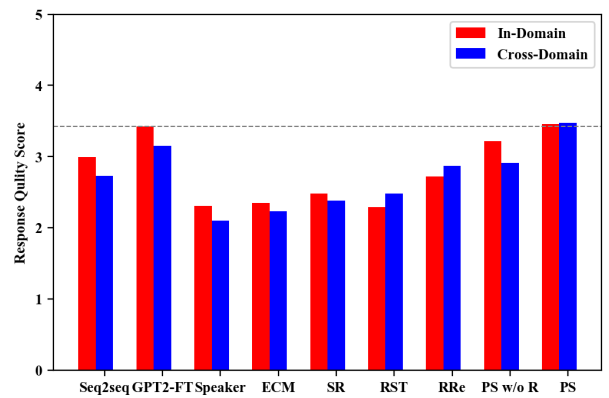

Figure 1: In-domain and cross-domain evaluations on the quality of the generated responses. The red column represents the averaged quality score on in-domain test set, and the blue column denotes the averaged quality score after domain variation.

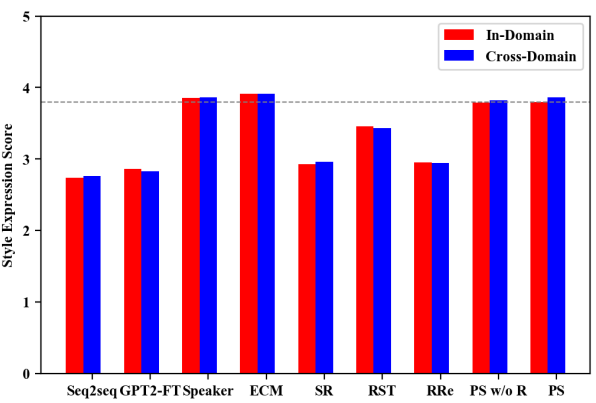

Figure 2: In-domain and cross-domain evaluations on the style expression of the generated responses. The red column represents the averaged ranking on in-domain test set, and the blue column denotes the averaged style expression score after domain variation.

between the two different domains. The same effect can also be observed in the results of RST and RRe which also use the retrieved results and get a even higher performance when facing domain variation.

Secondly, looking at style expression performance in Figure 2, we can see that there is not obvious difference between the results of in-domain and cross-domain evaluations. Our analysis is that, to generate responses with desired language style, the model could simply generate the characteristic expressions for that language style without considering the input query. Therefore, the domain variation actually poses 
ACL 2020 Submission ${ }^{\star \star \star}$. Confidential Review Copy. DO NOT DISTRIBUTE.

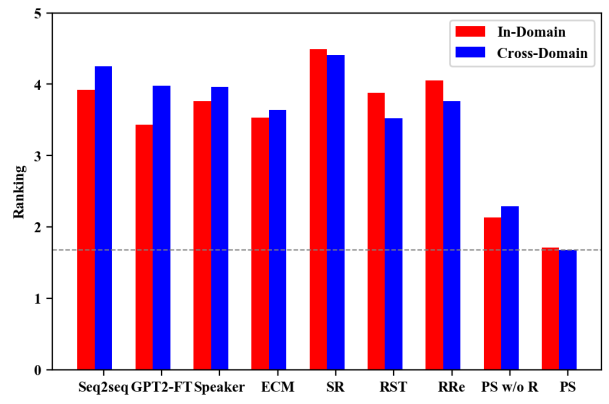

Figure 3: In-domain and cross-domain evaluations on the ranking of generated responses. The red column represents the averaged ranking on in-domain test set, and the blue column denotes the averaged ranking after domain variation.

Finally, by jointly considering the quality and style expression, from Figure 3 we can see that the proposed model achieves best ranking for both in-domain and cross-domain evaluation. Therefore, it is safe to say that the proposed model is the best and the most robust one among all approaches. 